\title{
EFFICIENT AND PRACTICAL HANDLING OF SPATIO-TEMPORAL DATA BASED ON TIME-DEPENDENT NET COMPONENTS
}

\author{
P. V. Kuper ${ }^{1 *}$ \\ ${ }^{1}$ Geodetic Institute, Karlsruhe Institute of Technology, Germany - kuper@kit.edu
}

Commission IV, WG IV/7

KEY WORDS: Geo-Data Management, Spatio-Temporal Data, Geodatabase, 3D/4D GIS

\begin{abstract}
:
The monitoring of spatio-temporal phenomena such as the movement of glaciers or volcanos produces huge amounts of data. Especially the handling of time as a fourth dimension usually requires terabytes of storage space. Therefore, an appropriate data management model for an efficient handling of such data is required and a key concept for any 3D/4D GIS. Within this paper, a comprehensive data management model is presented that optimizes the handling and storage of spatio-temporal data. The concept of time-dependent Net Components is introduced to handle regions that are monitored and modelled in individual time steps. Combined with the concept of Point Tubes the resulting data management model is used to manage spatio-temporal data based on d-simplicial complexes with dimensions $\mathrm{d} \epsilon\{0,1,2,3\}$. It is shown that the model is capable of handling various kinds of spatiotemporal applications. These include the proper handling of variable temporal discretizations of partial regions within a 4D model. The storage requirements are reduced and spatial as well as spatio-temporal operations are accelerated significantly. Critical issues such as the preservation of the net topology between time steps and the handling of boundary regions of adjacent net components can be solved.
\end{abstract}

\section{INTRODUCTION}

The model of simplicial complexes can be used for the geometric modelling of natural structures, e.g. the earth's surface and geological subsurface (Breunig et al., 1999; Mallet, 2002; Xing et al., 2015). In many applications it is necessary to consider a temporal aspect, i.e. to describe the transformation of a certain region within time. An important focus of research is the modelling of 3D spatio-temporal data that have their origin in the field of earth sciences, particularly geology, geothermal energy and geomorphology.

When 3D data gets extended by a temporal component it is possible to model the continuous transformation of natural structures such as soil layers (Weihed, 2015). Multiple modelling tools such as GOCAD ${ }^{\circledR}$, GeoModeller ${ }^{\circledR}$ or Petrel ${ }^{\circledR}$ are used for the development of digital 3D models. This paper focuses on models that consist of d-simplicial complexes with a dimension $d \in\{0,1,2,3\}$. Accordingly, point clouds, segment-, triangle- and tetrahedra-nets form the geometric basis to describe natural structures such as a glacier, a geologic layer or an active volcano (Mallet, 1992, Mallet, 2002). The temporal transformation of such structures is realized by time series that consist of interrelated 3D models, cf. Figure 1.

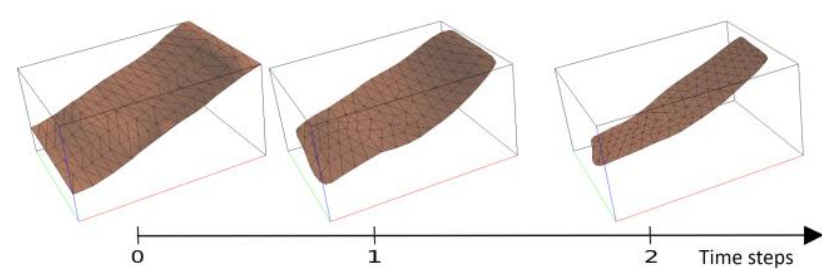

Figure 1. 4D model composed of three 3D models, each representing a different time step

Professional geodatabases provide a comfortable data management including a support of various spatial access methods. According to (Worboys and Duckham, 2004) a geodatabase forms the heart of any GIS. Geodatabases that handle time as an additional dimension need to meet a significantly extended set of requirements and are subject of ongoing research (Le, 2014; Gabriel et al., 2015; Ohori, 2016; Breunig et al., 2016).

The support of a temporal component for simplex based data within conventional modelling tools is still limited and in development (Mejia et al., 2015; Royer et al., 2015). Therefore, the composition and handling of 4D models is one important task for a 3D/4D geodatabase. By linking of 3D models to an explicit 4D model, continuously changing phenomena can be managed. Thus, the access to individual time steps of the model is facilitated. Additional intermediate steps may be calculated with the help of interpolation routines. Due to this procedure we can model time-continuous phenomena.

When dealing with spatio-temporal phenomena we need to handle huge amounts of data. Therefore, it is essential to improve the internal management by the development of 
appropriate data structures and data handling concepts, i.e. a data management model. Such a model must be suitable for an implementation within a spatial database.

\section{RELATED WORK}

Among others, (Polthier and Rumpf, 1994; Shumilov et al., 2002; Siebeck, 2003; Worboys and Duckham, 2004; Breunig et al., 2009; Le, 2014) have developed multiple concepts for the management of spatio-temporal data. Although 2D data is commonly used for conventional map applications, the field of $3 \mathrm{D}$ vector data becomes increasingly relevant (Breunig and Zlatanova, 2011; Pouliot et al., 2013; Gabriel et al., 2015; Biljecki et al., 2015).

Spatial data can contain various information in addition to purely geometric attributes. These include, for instance, thematic or semantic data (e.g. soil quality) or meta-data (e.g. date of the survey). In addition, spatial data, regardless if $2 \mathrm{D}$ or $3 \mathrm{D}$, can be expanded to a higher dimension to include a temporal component.

In the case of natural forms it is most common to use the data model of simplicial complexes, for soil layers or any kind of natural surfaces (Mallet, 2002). A simplicial complex is constructed by the combination of simplices. A d-simplex is the minimal object within the respective spatial dimension d. A formal definition is given by Spanier (1966). Worboys (1992) proposes the term of a d-simplicial complex whereas all simplices of a simplicial complex are of the same dimension $\mathrm{d}$. This follows the observation that the modelling of natural objects in most cases consist of simplices of just one dimension which form such a d-simplicial complex (Bär, 2007). For instance, a 2-simplicial complex is a simplicial complex that consists exclusively of 2-simplices. Such d-simplicial complexes are usually placed in a three-dimensional Euclidean space.

Within a d-simplicial complex d-simplices can share border regions which consist of (d-1)-simplices, i.e. corresponding to the case of two adjacent 2-simplices there exist only one shared 1-simplex, cf. Figure 2.

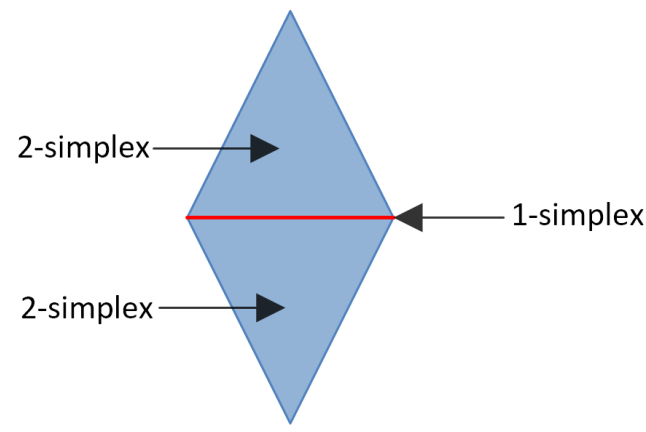

Figure 2. Two 2-simplices that share a segment (1-simplex) and form a 2-simplicial complex

The model of simplicial complexes forms the foundation of the spatio-temporal data model which is used in this paper. For the modelling of time-continuous phenomena (e.g. the development of Dubai within the last 50 years), time needs to be introduced as a new dimension and handled accordingly. For instance, geological changes are usually modelled in continuous processes. However, when modelling the development of buildings or entire cities, discrete changes are in the focus of interest and must be specified accordingly (Oosterom and Stoter, 2010).

In this paper a spatio-temporal object is referred to as $4 \mathrm{D}$ object or 4D model and consists of several 3D models representing individual time steps, cf. Figure 1. This approach for the composition of spatio-temporal data is referred to as snapshot model (Langran and Chrisman, 1988; Renolen, 1997). In this case time is regarded as continuous.

For the modelling of continuous phenomena, huge amounts of spatio-temporal data are generated. A 3D model that represent a single time step of a 4D model usually consists of more than 100.000 net elements (Hu et al., 2010; Xing et al., 2015). Since a 4D model consists of multiple of such time steps, there are high demands on the storage capacities. Operations such as translations, interpolations or intersections between models, require lots of computing power. Therefore, we present a data management concept that optimizes the handling and storage of spatio-temporal data with partial regions that are monitored and modelled by individual time steps.

The data management model presented in this paper is based on two concepts: the concept of Point Tubes and the concept of Net Components. The Point Tube concept separates the net topology from the vertices of a d-simplicial complex (Breunig et al., 2013a; Kuper et al., 2016; Breunig et al., 2016). These vertices are managed in so-called Point Tubes, a special time dependent data structure. The concept of Net Components is used to manage spatio-temporal data with regions that are monitored and modelled in individual time steps (Kuper, 2010). The adjustments of the concepts for the handling of d-simplicial complexes with dimensions $d \in\{0,1,2,3\}$ and their consolidation are described in detail in the following section.

\section{A CONSOLIDATED DATA MANAGEMENT MODEL} FOR SPATIO-TEMPORAL DATA

The spatio-temporal data management model of this paper describes the data handling for continuous processes, e.g. a continuous movement. Referring to (Worboys, 1994), such a process is represented by discrete time steps. In the temporal dimension these time steps form the support points of a 4D model. Accordingly, each time step of a $4 \mathrm{D}$ model is a 3D model that consists of an amount of $d$ simplices which form a d-simplicial complex with $d \in\{0,1,2,3\}$. Partly due to improving recording methods such $3 \mathrm{D}$ models consist of vast amounts of data (Hu et al., 2010; Xing et al., 2015). When storing or processing such data, an optimization of the performance and the reduction of storage requirements is of fundamental importance.

First, the requirements of a data management model for spatiotemporal data are elaborated. The following list presents a summarized overview of such requirements:

- Support of subregions with individual time steps.

- Optimization of calculation processes and avoidance of unnecessary operations.

- Optimization of storage demands.

- Foundation for the implementation of advanced spatiotemporal analysis functions.

- Linking of individual 3D discrete time steps into a continuous 4D model (3D + time). 
- Option for an interpolation between time steps.

When continuous movements of structures are modelled within a 4D model, subregions might behave more volatile than others. Such a subregion can be e.g. a landfill within a mountain area, cf. Figure 3. To satisfy such a process within a data management model, so-called net components are introduced. Due to this concept a division of 4D models in subregions with different temporal resolutions is modelled appropriately. This concept is combined with the concept of Point Tubes which is presented in the following.

\subsection{Point Tube Concept}

According to Egenhofer et al. (1990) the vertices within a simplicial complex are unique. Following this statement, the indexed-face-set procedure separates the vertices and the corresponding mesh. Common data formats, such as the OBJ format and the VRML format have adapted this procedure (Carey and Bell, 1997). This kind of separation was used by Siebeck (2003) and Le (2014) in the context of managing spatio-temporal data within a geodatabase.

Due to the separation of the vertices from the net topology, the following benefits occur:

1. The net topology (mesh) of a model can be reused across multiple time steps.

2. Vertices which are used by multiple elements are stored uniquely.

Based upon this separation the vertices of a net are managed in a special data structure, the so-called Point Tubes (Rolfs, 2005; Breunig et al., 2013a; Kuper et al., 2016; Breunig et al., 2016). Thus, the movement of these vertices in space is clearly assigned and can be tracked over time. For an appropriate response to a request of a specific time step of the 4D model, the net topology is combined with the Point Tube data structure. An appropriate 3D model can be created for any point in time, once the date is within the time interval of the $4 \mathrm{D}$ model. For this propose, the vertices for the result object are calculated by the use of interpolation of all involved Point Tubes.

\subsection{Handling of Multiple Temporal Discretizations}

4D models usually reflect continuous processes. Such a continuous process is realized by $3 \mathrm{D}$ models which refer to individual time steps. Complex 4D models may consist of subregions, which have different temporal resolutions. For instance, if the subject of interest is the continuous movement of a glacier we observe an active subregion on top of an almost stationary basis. We observe the same characteristics when modelling an area with an active volcano. The monitoring of such a subregion would be intensified to map the volatile movement within this particular area. Thus, the temporal resolution has increased for this region, cf. Figure 3.

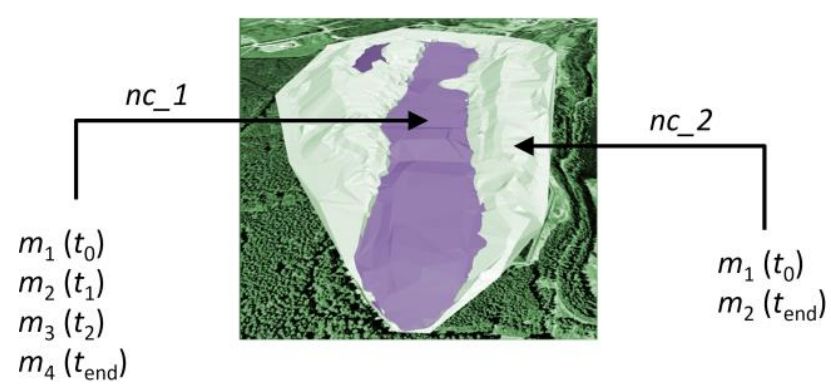

Figure 3. Different temporal discretizations for subregions of a 4D model (based on the Piesberg dataset by Lautenbach and Berlekamp (2002))

The data management model must provide the ability to handle such parts of a 4D model with different temporal resolutions to cover such processes. Therefore, contiguous regions with the same temporal resolution are summarized within an individual net component. The entire 4D model is composed by a combination of all involved net components. From an outside perspective, the 4D model changes continuously as intermediate steps can be interpolated. For the usage of net components certain conditions must be met:

- Within a net component, solely the geometry changes, the net topology remains unchanged.

- The net topology at the border regions of two adjacent net components must remain consistent over the entire time interval of the 4D model.

- The time interval $\left(\mathrm{t}_{0}, \mathrm{t}_{\mathrm{end}}\right)$ of all net components of a $4 \mathrm{D}$ model must be uniform.

The concept of net components has been studied and implemented for spatio-temporal data based on triangle nets, i.e. 2-simplicial complexes (Kuper, 2010). Within this paper the concept is extended to the net dimensions $d \in\{0,1,2,3\}$.

\subsubsection{Realization for 0D-nets}

Since the concept of different time discretizations for subregions was initially developed in combination with the Point Tube concept, the transfer to handle 0D-nets is trivial. The vertices of each 0D-net component consist of Point Tubes that represent the temporal resolution of the particular component, cf. Figure 4.

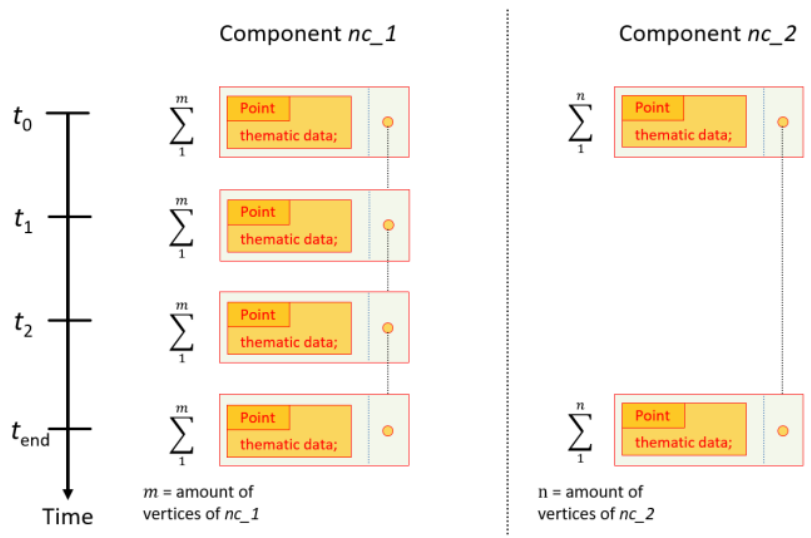

Figure 4. Internal structure representing the management of a OD-net, which is composed of two components with different temporal resolutions 
Due to the lack of a net topology, the Point Tubes from the border region of a $0 \mathrm{D}$-net component can be of the same temporal resolution as the corresponding component these points belong to. There are no overlapping regions and different net components are completely separated from each other.

\subsubsection{Realization for 1D-nets}

The concept of different temporal discretizations for net components can be applied to the management of 4D models that consist of 1D-nets, i.e. segment nets. If subregions of such a model are based on different temporal discretization, these parts are aggregated within a net component. Figure 5 shows a 1D-net that consists of two net components $n c_{-} l$ and $n c_{-} 2$ with different temporal resolutions.
Component nc_1
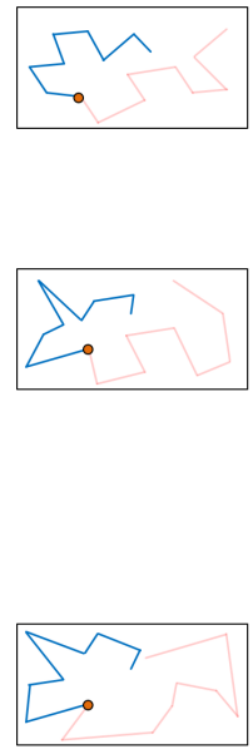

○ = border region

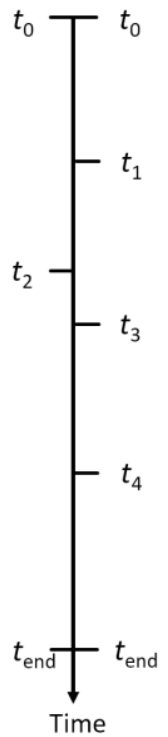

Time
Component nc_1
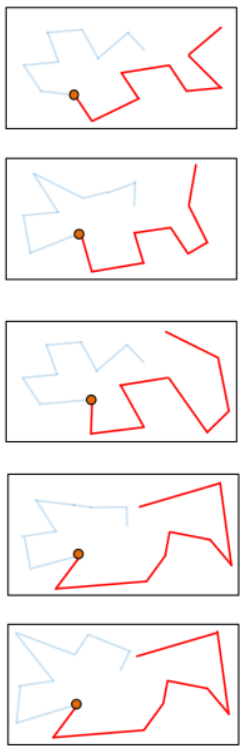

0 = border region
Figure 5. 1D-net consisting of two net components with different temporal resolutions, i.e. net component $n c_{-} l$ with 3 time steps, net component $n c \_2$ with 5 time steps

\subsubsection{Realization for 2D-nets}

The concept of net components with different temporal discretizations was already combined with the Point Tube concept by Kuper (2010). In this case Point Tube data structures belong to a particular net component and share their temporal resolution.

The border regions of such net components must be treated with special attention. Otherwise inconsistencies in the net topology might appear. The border regions must remain consistent even during interpolation operations between the time steps. For this issue two different approaches are presented in the following. The first approach focuses on the border region and postulates that in two adjacent net components, the time steps of one net component must be a refinement of the temporal discretization of the other component. This is e.g. the case when the net component $n c_{-} l$ consists of the time steps $m l\left(\mathrm{t}_{0}\right), m 2\left(\mathrm{t}_{1}\right)$ and $m 3\left(\mathrm{t}_{2}\right)$ and the net component $n c \_2$ of the time steps $m 1\left(\mathrm{t}_{0}\right)$ and $m 2\left(\mathrm{t}_{2}\right)$. In addition, the shared vertices of such net components must be on a common line during the interpolation process, cf. Figure 6.

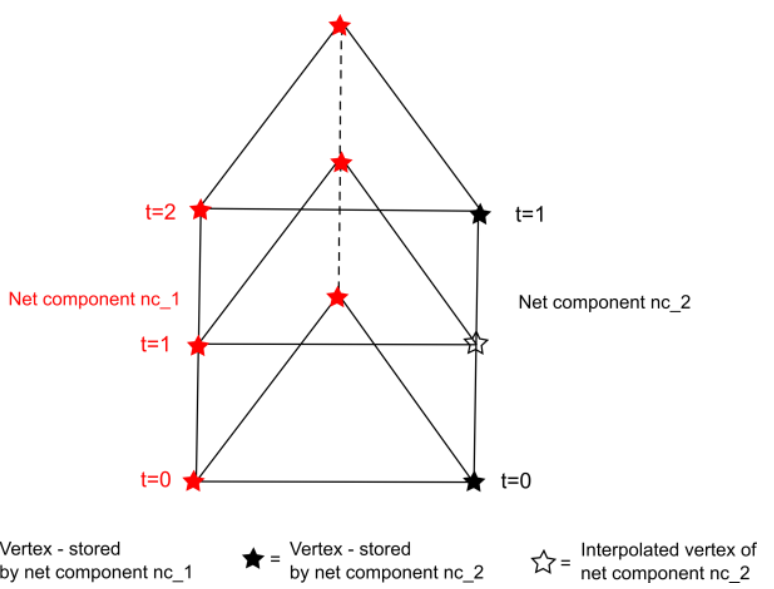

Figure 6. Spatio-temporal prism representing a shared border region

Due to these conditions the border regions of adjacent net components remain consistent and valid across the entire time interval. In addition, with this approach the border region of net component $n c \_2$ with the lower temporal resolution remains independent of $n c_{-} l$ between time steps $\mathrm{t}_{0}$ and $\mathrm{t}_{2}$.

The second approach proposes the introduction of so-called hybrid elements and focuses on the vertices of a border region between two net components. This procedure eliminates the condition that the temporal discretization of the involved net components must be a refinement of ones another. The vertices of the border region from the component with the finer discretization must no longer be on the trajectory, which is created by the interpolation between two time steps of the coarser component, cf. Figure 7.

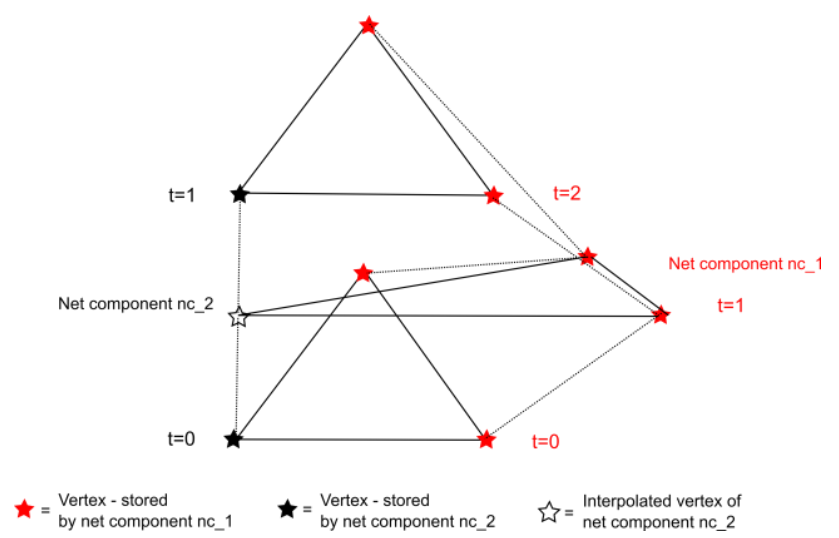

Figure 7. Spatio-temporal prism representing a shared border region with hybrid elements

However, it must be ensured that no data points of the border region enter the boundary of uninvolved net elements. Otherwise this could destroy the topology of the net.

Due to this concept, it is possible to handle different temporal discretizations of subregions within a 4D model. This leads to a major saving of storage space, since rapidly changing parts of a 4D model are handled accordingly while the rest of the model can be managed in a lower temporal resolution. Figure 8 shows the Point Tube data structure of a 4D model that consists of two 
net components $n c \_l$ and $n c \_2$ with time steps $m l\left(\mathrm{t}_{0}\right), m 2\left(\mathrm{t}_{1}\right)$, $m 3$ ( $\left.\mathrm{t}_{2}\right), m 4$ (tend) and $m 1$ ( $\left.\mathrm{t}_{0}\right), m 2$ (tend), respectively.

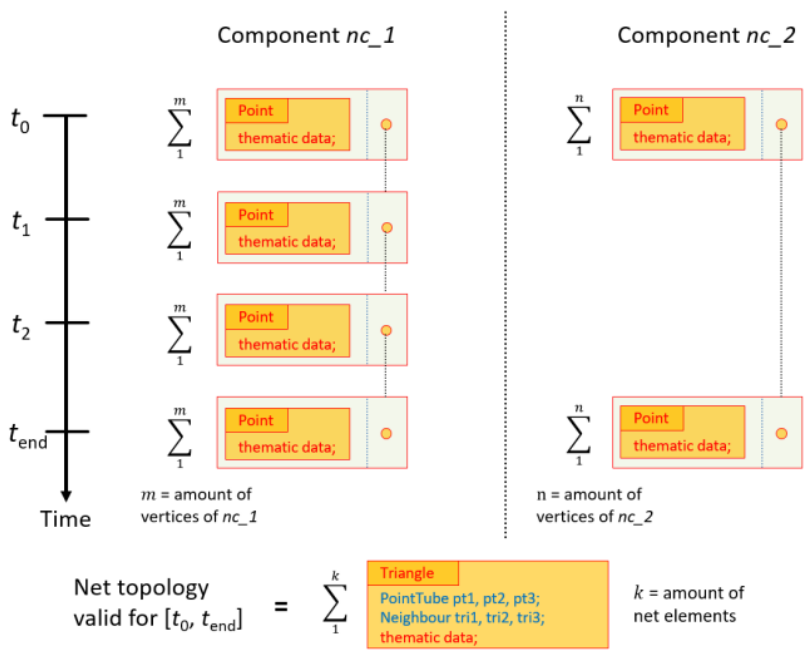

Figure 8. Point Tube data structure for two net components with different temporal discretizations

Due to the combination of this approach with the concept of Point Tubes a solution for maintaining the consistency of border regions of adjacent net components is given.

\subsubsection{Realization for 3D-nets}

The concept can be applied on 4D models that consist of 3Dnets, i.e. tetrahedron nets. The procedure is analogously to the management of 2D-nets. However, the realization of those conditions for the border regions may be much more challenging. It must be ensured that parts of the border region do not penetrate net elements, i.e. tetrahedra, of other net components, cf. Figure 9. This condition is especially critical when a net component is embedded within another one.

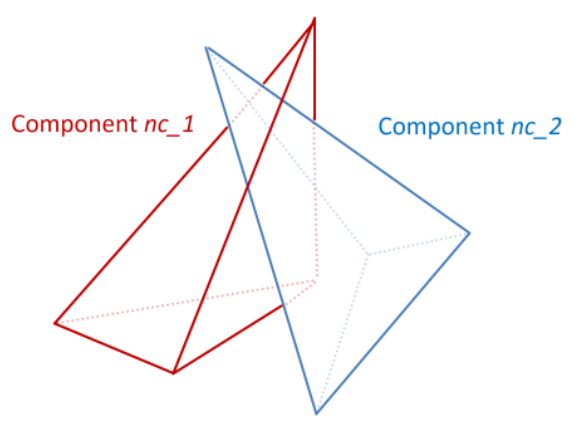

Figure 9. Violation of the net topology consistency due to modifications at the border region between two 3D-net components with different temporal discretisations

The management of such border regions is realized with the help of Point Tubes. The net topology of the border region itself must be kept in a consistent state according to the rules of the Point Tube concept. When managing adjacent 3D-net components with different temporal resolutions, this problem should be significantly reduced compared to embedded ones.

If an explicit model $m\left(t_{\mathrm{x}}\right)$ for the specific time $\mathrm{t}_{\mathrm{x}}$ is required during a range query or a geometric operation, such a model is formed by the union of all involved net components. If an explicit time step was stored for time $t_{x} \in\left(t_{s t a r t}, t_{e n d}\right)$ for one or more net components, this specific time step is used for the construction of $m\left(\mathrm{t}_{\mathrm{x}}\right)$. All other net components are created by an interpolation for time $t_{x}$. This procedure applies to all net dimensions.

Net components with different temporal discretizations support the management of 4D models with different volatile subregions. One important issue is the consistency of the time intervals of all involved net components. All net components of a 4D model must be valid within the entire corresponding time interval. The individual Point Tubes contain the vertices of the respective net component in the respective temporal discretization. For the border regions between such net components a sharing of net elements only occurs in higher dimensional structures, starting from 1-simplicial complexes. In this case the vertices are immutable assigned to a fixed net component.

\section{DISCUSSION}

The most important positive effects of the comprehensive data management model compared to a conventional approach are:

\section{Reduction of storage space}

By separating the net topology from the vertices of a 4D model it is possible to save storage space, since the net topology is reused for multiple time steps. Furthermore, due to the realization of the indexed-face-set procedure the multiple storage of vertices at net elements is avoided. Thus the completeness axioms are respected, and storage space is saved when managing 4D models based on d-simplicial complexes with $d \in\{1,2,3\}$. Due to the introduction of net components with various temporal discretizations different volatile subregions of a 4D model are treated accordingly. Therefore, it is possible to avoid the storage of the entire model in a high frequency of time steps, which leads to an elimination of entire time steps for suitable subregions.

\section{Acceleration of spatial and spatio-temporal operations}

Due to the introduction of Point Tube data structures multiple calculations, such as the translation of a 4D object, must be performed exclusively for the vertices. These are separated from the net topology and can be accessed without the use of net elements. Vertices that are used by multiple net elements (within 1-3D-nets) are treated uniquely, which accelerates the processing of operations. In addition, the use of the Point Tube concept obtains the consistency of the net topology as the mesh is resistant against possibly arithmetic errors.

\section{Extended field of applications}

The concept for handling different temporal discretizations of partial regions extends the range of possible applications. Due to this concept it is possible to manage $4 \mathrm{D}$ models that consist of multiple regions with different volatile movements. This is the case, e.g. once a region changes faster than other regions and the monitoring is focused on this particular area. Due to this concept, it is possible to embed such regions in an appropriate spatio-temporal context. The entire 4D model is managed collectively in a single entity.

The reduction of the storage requirements by the use of the Point Tube concept is only relevant for $4 \mathrm{D}$ models that are 
based on $\mathrm{d}$ simplicial complexes with $\mathrm{d} \in\{1,2,3\}$, as 0 -simplicial complexes do not have net elements that share vertices. Nevertheless, by the unambiguous assignment of vertices across the time steps of a $4 \mathrm{D}$ model, some important operations are supported, such as the interpolation between two time steps. This benefit applies on all net dimensions. Such operations benefit not only from the unambiguous structure of the individual vertices across different time steps, but also from the cumulative access to all vertices of a 4D model. The concept of net components with different temporal discretizations does not require any additional resources once it is not needed. In such a case, the entire net consists of one single component. Therefore, only positive effects are expected.

\subsection{Constraints and Limitations}

The presented data management model is suitable for 4D models that meet the conditions that were previously outlined. Additionally, there are constrains that need to be met by an appropriate 4D model for a correct behaviour of the comprehensive model. These partly depend on the dimension of the particular net. The handling of 0 -simplicial complexes is uncritical, but for d-simplicial complexes with $d \in\{1,2,3\}$ it must be assured that the structure of the simplicial complex is valid at any time. One key issue is the compliance of neighbourhood information of the net elements. A change of such information would result in a violation of the simplicial complex structure within a time interval.

Another important constraint that refers to the compliance of the simplicial complex structure is a correct behaviour of border regions. If a $4 \mathrm{D}$ model consists of multiple net components the net topology must be in a consistent state within the entire time interval. An implementation of the comprehensive data management model might report a violation but cannot solve it automatically. In practice such constrains are already respected during the construction of a single time step, i.e. a 3D model. This is partly due to the constraints which are imposed by $3 \mathrm{D}$ modelling tools such as GOCAD®.

\section{OUTLOOK}

In the future the data management model will be tested with additional data sets. Landslides and volcano applications of our geoscientific project partners seem to be suitable and promising candidates.

The integration of further components into the workflow could, for instance, include 3D/4D modelling tools such as GOCAD ${ }^{\circledR}$ as a conventional client application. Other promising components are virtual reality (VR) devices such as Oculus Rift ${ }^{\circledR}$ or HTC Vive ${ }^{\circledR}$. Appropriate data can be prepared within a 3D/4D geodatabase architecture that is based on the presented data management model. Due to the capabilities of VR devices the user can experience a real 3D impression of the models. A real-time interpolation between individual time steps of $4 \mathrm{D}$ models could improve the visualization of particular changes within a 4D model. Due to a cable connection to a conventional desktop PC the required computation power should be available.

Another direction of further developments is the realization of advanced spatio-temporal analysis functions. Such functions can include, for instance, the calculation of differences of a geometric model between two time steps of a 4D model or the prediction of a transformation for future dates.
As pointed out, there are several advanced query facilities which can be used in different application fields. Further investigations can focus on the detection of additional use cases. The access to these facilities, e.g. based on an implementation on top of a geodatabase architecture, should be designed in a user-friendly way, i.e. a simple access should be provided.

\section{ACKNOWLEDGEMENTS}

The work presented in this paper is mainly based on the results of the authors PhD thesis (Kuper, 2016).

\section{REFERENCES}

Bär W., 2007. Management of geo-scientific 3D data in mobile database systems, Ph.D. Thesis, University of Osnabrück, Germany, 166p.

Biljecki F., Stoter J., Ledoux H., Zlatanova S., Çöltekin A., 2015. Applications of 3D City Models: State of the Art Review, In: ISPRS International Journal of Geo-Information, pp. 2842 2889. doi=https://doi.org/10.3390/ijgi4042842.

Breunig M., Zlatanova S., 2011. 3D geo-database research: retrospective and future directions. In: Computers \& Geosciences, Vol. 37(7), pp. 791-803. doi=https://doi.org/10.1016/j.cageo.2010.04.016.

Breunig M., Cremers A.B., Götze H.-J., Schmidt S., Seidemann R., Shumilov S., Siehl A., 1999. First steps towards an interoperable 3D GIS - an example form southern Lower Saxony, Germany, In: Physics and Chemistry of the Earth, Part A, Vol. 24(3), pp. 179-190.

Breunig M., Schilberg B., Thomsen A., Kuper P.V., Jahn M., Butwilowski E., 2009. Towards a 3D/4D geo-database supporting the analysis and early warning of landslides, In: Proceedings of Cartography and Geoinformatics in Early Warning and Crises Management - towards better solutions, $17 \mathrm{p}$.

Breunig M., Butwilowski E., Golovko D., Kuper P.V., Menninghaus M., Thomsen A., 2013. Advancing DB4GeO, In: Progress and New Trends in $3 D$ Geoinformation Sciences, Lecture Notes in Geoinformation and Cartography, Springer, pp. 193-210.

Breunig M., Kuper P.V., Butwilowski E., Thomsen A., Jahn M., Dittrich A., Al-Doori M., Golovko D., Menninghaus M., 2016. The Story of DB4GeO - A Service-Based Geodatabase Architec-ture to Support Multi-Dimensional Data Analysis and Visualization, In: ISPRS Journal of Photogrammetry and Remote Sensing, Volume 117, pp. 187-205.

Carey R., Bell G., 1997. The Annotated VRML 2.0 Reference Manual, Essex, UK, Addison-Wesley Longman Ltd., 1997.

Egenhofer M.J., Frank A. U., Jackson J. P., 1990. A Topological Data Model for Spatial Databases, In: Proceedings of the First Symposium on Design and Implementation of Large Spatial Databases, New York, NY, USA, pp. 271-286. 
Gabriel P., Gietzel J., Le H.H., Schaeben H., 2015. GST: A Network Based Datastore for Geoscience Data and Geomodels and Its Implementation-ProMine's Contribution Towards Interoperability, In: 3D, 4D and Predictive Modelling of Major Mineral Belts in Europe, P. Weihed, Ed. Springer International Publishing, pp. 59-71.

Hu L., Sander P. V., Hoppe H., 2010. Parallel view-dependent level-of-detail control, In: IEEE Trans Vis Comput Graph, Vol. 16 , no. 5 , pp. $718-728$.

Kuper, P.V., Breunig, M., Al-Doori, M., Thomsen, A., 2016. Application of 3D Geospatial Plus Temporal Data Modeling, Management, and Analysis in DB4GeO, In: ISPRS Ann. Photogramm. Remote Sens. Spatial Inf. Sci., Vol. IV-2/W1, 163-170, doi:10.5194/isprs-annals-IV-2-W1-163-2016, 8p.

Kuper, P.V., 2010. Entwicklung einer 4D Objekt-Verwaltung für die Geodatenbank DB4GeO, Diploma thesis, University of Osnabrück, 64p.

Kuper, P.V., 2016. Spatio-Temporal Data Handling for Generic Mobile Geoinformation Systems, PhD thesis, 189p.

Langran G., Chrisman N.R., 1988. A Framework for Temporal Geographic Information, In: Cartographica, Vol 25(3), pp. 114.

Lautenbach S., Berlekamp J., 2002. Datensatz zur Visualisierung der Zentraldeponie Piesberg in Osnabrück, University of Osnabrück.

Le H.H., 2014. Spatio-temporal Information System for the Geosciences: Concepts, Data models, Software, and Applications, $\mathrm{PhD}$ thesis, Technische Universität Bergakademie Freiberg, 169p.

Mallet J.L., 1992. GOCAD: a Computer Aided Design Programme for Geological Applications, Turner A.K. (Ed.), Three-Dimensional Modelling with Geoscientific Information Systems. In: Proceedings of NATO ASI 354, Kluwer Academic Publishers, Dordrecht, pp. 123-142.

Mallet J.L., 2002. Geomodeling, Oxford University Press, 599 p.

Mejia P., Royer J. J., Fraboulet J. G., Zielińska A., 2015. 4D Geomodelling A Tool for Exploration-The Kupferschiefer in The Lubin Region, Poland, In: $3 D, 4 D$ and Predictive Modelling of Major Mineral Belts in Europe, Mineral Resource Reviews, pp 163-187.

Ohori K.A., 2016. Higher-dimensional modelling of geographic information, PhD thesis, Delft University of Technology, 236p.

Van Oosterom P., Stoter J., 2010. 5D Data Modelling: Full Integration of 2D/3D Space, Time and Scale Dimensions, In: Proceedings of the 6th International Conference on Geographic Information Science, Berlin, Heidelberg, pp. 310 324.

Penninga F., 2008. 3D Topography. A Simplicial Complexbased Solution in a Spatial DBMS, PhD thesis, In: Publications on Geodesy 66, TU Delft, Netherlands, 204p.
Polthier K., Rumpf M., 1994. A concept for Time-Dependent Processes, In: Goebel et al., (Eds), Visualization in Scientific Computing, Springer, Vienna, pp. 137-153.

Pouliot J., Daniel S., Hubert F., Zamyadi A. Eds., 2013. Progress and New Trends in 3D Geoinformation Sciences, 2013 edition. Heidelberg, New York: Springer, 314p.

Renolen A., 1997. Temporal Maps and Temporal Geographical Information Systems (Review of Research), Department of Surveying and Mapping, The Norwegian Institute of Technology, 12p.

Rolfs C., 2005. Konzeption und Implementierung eines Datenmodells zur Verwaltung von zeitabhängigen 3D-Modellen in geowissenschaftlichen Anwendungen, Diploma thesis, 90p.

Royer J.J., Mejia P., Caumon G., Collon P., 2015. 3D and 4D Geomodelling Applied to Mineral Resources Exploration - An Introduction, In: $3 D, 4 D$ and Predictive Modelling of Major Mineral Belts in Europe, Mineral Resource Reviews, pp. 73-89.

Shumilov S., Thomsen A., Cremers A.B., Koos, B., 2002. Management and visualisation of large, complex and timedependent 3D objects in distributed GIS, In: Proceedings of the Tenth ACM International Symposium on Advances in Geographic Information Systems, pp. 113-118.

Siebeck J., 2003. Concepts for the Representation, Storage, and Retrieval of Spatio-Temporal Objects in 3D/4D GeoInformation-Systems, $\mathrm{PhD}$ Thesis, Faculty of Mathematics and Naturlal Sciences at the Rheinischen Friedrich-WilhelmsUniversity, Bonn, 143p.

Spanier, E.H., 1966. Algebraic topology, McGraw-Hill, 528p.

Weihed P., 2015. 3D, 4D and Predictive Modelling of Major Mineral Belts in Europe, 1st ed. 2015 edition. Springer, 330p.

Worboys M., Duckham M., 2004. GIS: A Computing Perspective, 2nd Edition. Boca Raton, FL, USA: CRC Press, Inc, 448p.

Worboys M., 1992. A generic model for planar geographical objects, In: International Journal of Geographical Information Systems, Vol. 6(5), pp. 353-372.

Worboys, M. F., 1994. Object-oriented approaches to georeferenced information, In: International Journal of Geographical Information Systems, Vol. 8(4), pp. 385-399.

Xing H., Liu Y., Gao J., Chen S., 2015. Recent development in numerical simulation of enhanced geothermal reservoirs, In: Journal of Earth Science, Vol. 26(1), pp. 28-36. 